\title{
Intraoperative Fluoroscopic Monitoring during TVM Surgery: Safer Procedure Even for Beginners
}

\author{
Hideki Kobayashi $^{{ }^{*}}$, Norifumi Sawada ${ }^{1}$, Satoru Kira ${ }^{1}$, Tatsuya Miyamoto ${ }^{1}$, Yaburu Haneda ${ }^{1}$, \\ Hidenori Zakoji ${ }^{1}$, Takayuki Tsuchida ${ }^{1}$, Isao Araki ${ }^{1,2}$, Masayuki Takeda ${ }^{1}$ \\ ${ }^{1}$ Department of Urology, University of Yamanashi, Chuo City, Japan \\ ${ }^{2}$ Department of Urology, Shiga University of Medical Science, Otsu, Japan \\ Email: hidekik@yamanashi.ac.jp
}

Received December 12, 2011; revised February 8, 2012; accepted February 15, 2012

\begin{abstract}
Tension-free vaginal mesh (TVM) surgery is a common and minimally invasive procedure for female pelvic organ prolapse. In 2004, this procedure was developed by a French group, and standardized surgical kits are now commercially available in many countries. Although it is less invasive, one of the shortcomings of this procedure is that it involves a single surgeon groping around with their fingers without any intraoperative monitoring. Therefore, using Intraoperative fluoroscopic monitoring during TVM surgery makes it safer, even for beginners. In this case, we performed TVM for the anterior vaginal wall. First, we used the c-arm of a fluoroscope to insert bilateral ureteral stents. A urethral catheter was then used for both urine drainage and contrast medium injection. In all procedures, we were able use fluoroscopic imaging whenever necessary. We were able to easily confirm the positions of the prolapsed bladder and the bilateral ureteral stents with fluoroscopic imaging, and the ischial spine was easy to locate before the procedure. We were also able to confirm the position of the top of the needle with fluoroscopic imaging whenever necessary. If a surgeon is worried about the risk of bladder injury during TVM surgery, they should inject contrast medium into the bladder at the start of the procedure. Intraoperative fluoroscopic monitoring during TVM surgery is easy and makes the procedure safer, even for beginners. Moreover, fluoroscopic imaging also allows intraoperative training. To avoid exposing the body to excess radiation, we must minimize the total length of the fluoroscopic examination.
\end{abstract}

Keywords: Pelvic Organ Prolapses; Surgery; Tension Free Vaginal Mesh; Fluoroscopic Imaging; Monitoring

\section{Introduction}

Tension-free vaginal mesh (TVM) surgery is a common and minimally invasive procedure for female pelvic organ prolapse [1-6]. In 2004, this procedure was developed by a French group [1], and standardized surgical kits are now commercially available in many countries. There are several kinds of modified procedure, each of which is designed for a particular type of prolapse. Although it is less invasive, one of the shortcomings of TVM surgery is that it involves a single surgeon employing a groping approach with their fingers without any intraoperative monitoring. Inevitably, there is always a risk of complications during surgery, including bladder, ureteral, bowel, vascular, and nerve injuries. In English literatures, it is reported that bladder injury rate is under a few percent [3-6]. In Japan, obtaining hands-on training using fresh cadavers is extremely difficult. Hence, blind techniques are always stressful, especially for beginners. Therefore, we have employed occasional intraoperative

${ }^{*}$ Corresponding author. fluoroscopic monitoring during TVM surgery, which has made the procedure safer, even for beginners.

\section{Procedure}

Patient: A 78-year-old woman with prolapse of the bladder, who underwent TVM for the anterior vaginal wall.

Surgical Procedure: All procedures were carried out with the patient under general anesthesia. The patient was placed on the operating table in a dorsosacral (lithotomy) position. First, we used the c-arm of a fluoroscope to insert bilateral ureteral stents $(6 \mathrm{Fr})$ by cystoscopy (Figure 1). This fluoroscope's c-arm is sometimes used during surgery. Using fluoroscopy, we were easily able to confirm the positions of the bilateral ureters, and grasping the prolapsed bladder wall allows us to gauge its distance from the bilateral ureters. The injection of epinephrine diluted with saline must be performed carefully through the bilateral course of the prolapsed bladder. The top of the Cathelin's needle ( $23 \mathrm{G}, 60 \mathrm{~mm}$ ) was located outside of the ureteral stents but inside the ischial spine. A urethral catheter is then used for urine drainage and contrast 
medium injection. Confirming the shape of the prolapsed bladder is very useful for improving the safety of TVM surgery. Detachment of the bladder from the vaginal wall is usually performed in a blind manner so we must take especial care during this procedure. The ischial spines are located on both sides of the bladder (Figure 2). After confirming the location of the arcus tendineus fascia pelvis (ATFP), we must drive a custom-made needle (Shimada style or Takeyama style) through the obturator foramen toward the ATFP. The first puncture is performed near to the obturator foramen (Figure 3), and the second puncture is performed near to the ischial spine (Figure 4). During all procedures, we were able to use fluoroscopic imaging whenever necessary.

\section{Discussion}

Pannu et al. are emphasizing the usefulness of fluoroscopic cystocolpoproctography for the diagnosis of pelvic

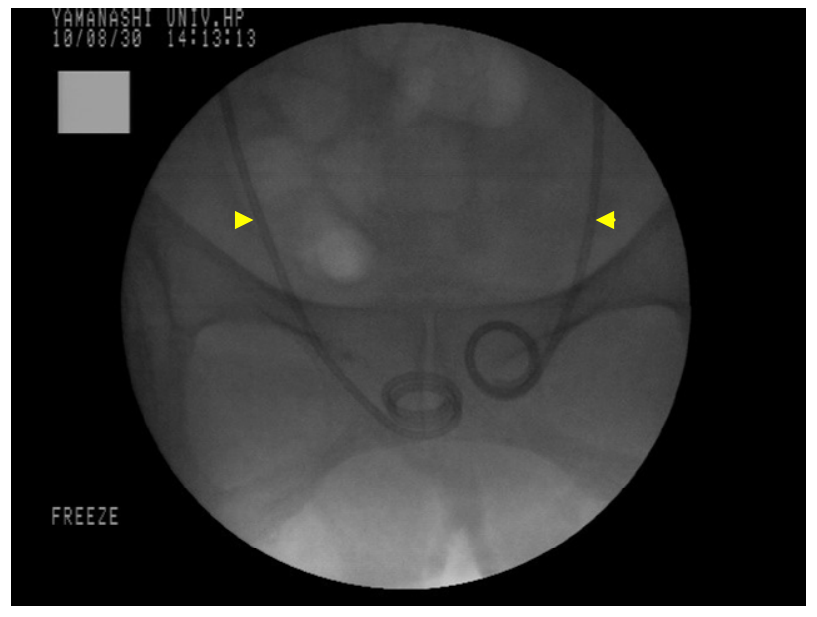

Figure1. The arrows indicate the both ureteral stents.

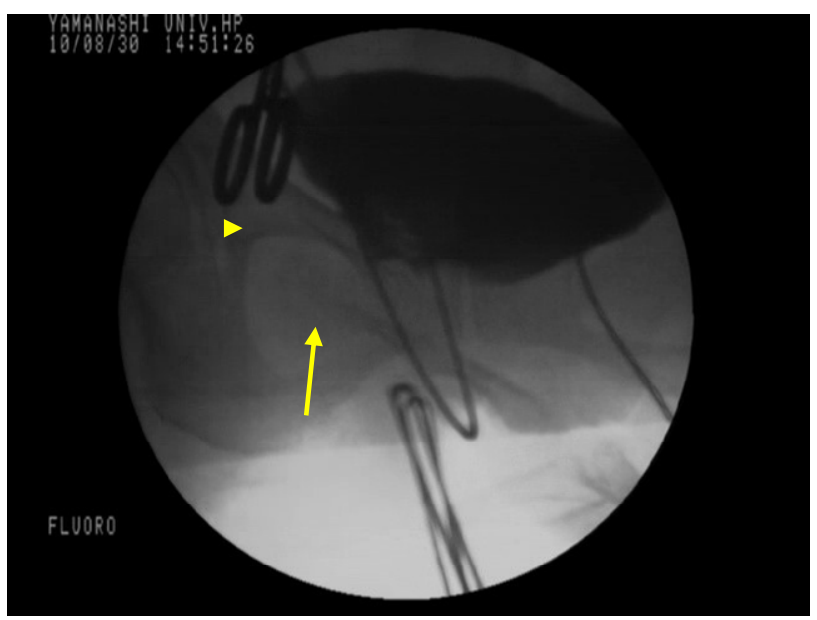

Figure 2. The bladder was visualized using contrast medium injected using a urethral catheter. Detachment of the bladder from the vaginal wall. The arrow indicates the finger bone, and the small triangle indicates the ischial spine.

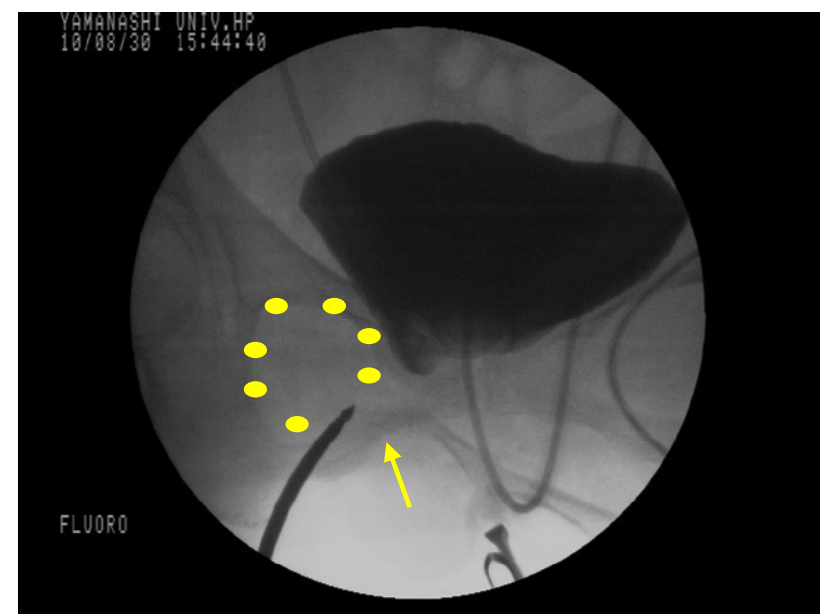

Figure 3. First puncture on the right side. The area surrounded by yellowish dots indicates the obturator foramen. The arrow indicates the finger bone.

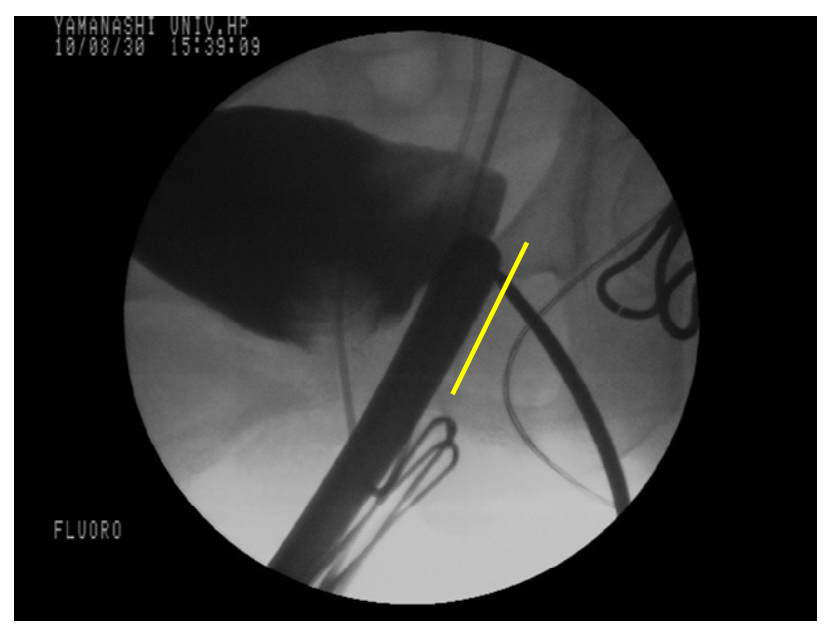

Figure 4. Second puncture on the left side. The yellowish line indicates the ATFP (arcus tendineus fascia pelvis). The spatula protects the bladder and ureter.

organ prolapse [7]. It was easy to confirm the position of the prolapsed bladder and bilateral ureteral stents with fluoroscopic imaging (Figures 1 and 2). The ischial spine was also easy to locate before the procedure. Moreover, we can use fluoroscopic imaging to teach young doctors technical points during the operation. In this case, we realized that the cervix was located relatively far from the bilateral ureters. We were able to confirm the position of the top of the needle (Cathelin's needle; $23 \mathrm{G}, 60 \mathrm{~mm}$ ) and custom-made needle (Shimada style or Takeyama style) with fluoroscopic imaging whenever necessary. Although the length of ATFP differs from patient, when the distance between the first and second puncture points is short, there is a possibility that the second puncture is not near the ischial spine, or otherwise the first puncture is not near the inside of obturator foramen. In fact, a reasonable distance and corresponding designed mesh is im- 
portant for good mesh positioning for each patient. If a surgeon is not afraid of ureteral injury, the ureteral stents are not necessary. On the other hand if a surgeon is worried about the risk of bladder injury during this procedure, they should inject contrast medium into the bladder at the start of the procedure. In such cases, it is sometimes necessary to drain urine from the bladder.

We experienced this fluoroscopic monitoring in five cases in all. In all cases, since we always reduced the cystocele before insertion of ureteral stents, kinking of ureter was recovered (in all cases, the insertion of ureteral stents was easy). The length of fluoroscopic monitoring differs from operator and cases. When the operator is beginner, the length of fluoroscopic monitoring might take a few minutes. From the second time, this procedure is not necessary or the length of monitoring is obviously less than the first time. Although, in our first case, we removed bilateral ureteral stents at the end of the surgery, the patient felt right back pain after the surgery. Ultrasound revealed bilateral slight hydronephrosis which was recovered in a few days. Since this event, we remove the ureteral stents after several days from the surgery. Another complication is hematuria. We experienced slight macroscopic hematuria in four cases out of five. These hematuria recovered easily by removing stents.

Although TVM surgery for pelvic organ prolapse is now common and is minimally invasive, the groping approach it involves is sometimes risky, especially for beginners, and can result in the neighboring organs being injured. So, we employed occasional intraoperative fluoroscopic monitoring during TVM surgery, which also made the training of young doctors safe and easy. Even for veteran surgeons, it is sometimes useful to employ fluoroscopic imaging to make certain procedures safer. To avoid exposing the body to excess radiation, we must minimize the length of the fluoroscopic examination. Although it is not necessary to employ fluoroscopic monitoring in all cases, this procedure is sometimes very useful, especially for risky cases such as hydronephrosis before reducing the prolapsed bladder, diverticulum in the bladder, pres- ence of previous operation for pelvic organ prolapsed, etc.

\section{REFERENCES}

[1] P. Debodinance, J. Berrocal, H. Clave, et al., "Changing Attitudes on the Surgical Treatment of Urogenital Prolapse: Birth of the Tension-Free Vaginal Mesh," Journal de Gynecologie Obstetrique et Biologie de la Reproduction, Vol. 33, No. 7, 2004, pp. 577-588. doi:10.1016/S0368-2315(04)96598-2

[2] M. Boukerrou, E. Lambaudie, P. Collinet, et al., "Objective Analysis of Mechanical Resistance of Tension-Free Devices," European Journal of Obstetrics \& Gynecology and Reproductive Biology, Vol. 124, No. 2, 2006, pp. 240-245. doi:10.1016/j.ejogrb.2005.06.040

[3] F. Caquant, P. Collinet, P. Debodinance, et al., "Safety of Trans Vaginal Mesh Procedure: Retrospective Study of 684 Patients," The Journal of Obstetrics and Gynaecology Research, Vol. 34, No. 4, 2008, pp. 449-456. doi:10.1111/j.1447-0756.2008.00820.x

[4] M. Takeyama, Y. Uesaka, S. Itoh, et al., "Feasibility of the Tension-Free Vaginal Mesh Procedure Using Soft Polypropylene Mesh (Gynemesh PS) Japan," International Urogynecology Journal and Pelvic Floor Dysfunction, Vol. 18, 2007, pp. 46-47.

[5] K. Kumiko, S. Shoji, Y. Shigeki, et al., "Clinical Pathway for Tension-Free Vaginal Mesh Procedure: Evaluation in 300 Patients with Pelvic Organ Prolapse," International Journal of Urology, Vol. 16, No. 3, 2009, pp. 314-317. doi:10.1111/j.1442-2042.2008.02249.x

[6] T. Satoru, O. Daisuke, S. Takahiro, et al., "Tension-Free Vaginal Mesh Procedure for Pelvic Organ Prolapse: A Single-Center Experience of 310 Cases with 1-Year Follow Up," International Journal of Urology, Vol. 17, No. 4, 2010, pp. 353-358. doi:10.1111/j.1442-2042.2010.02469.x

[7] H. K. Pannu, J. C. Scatarige and J. Eng, "Comparison of Supine Magnetic Resonance Imaging with and without Rectal Contrast to Fluoroscopic Cystocolpoproctography for the Diagnosis of Pelvic Organ Prolapse," Journal of Computer Assisted Tomography, Vol. 33, No. 1, 2009, pp. 125-130. doi:10.1097/RCT.0b013e318161d739 